\title{
Progression of Microstructural Damage in Spinocerebellar Ataxia Type 2: A Longitudinal DTI Study
}

\author{
M. Mascalchi, N. Toschi, M. Giannelli, A. Ginestroni, R. Della Nave, E. Nicolai, A. Bianchi, C. Tessa, E. Salvatore,
}

M. Aiello, A. Soricelli, and S. Diciotti

\begin{abstract}
BACKGROUND AND PURPOSE: The ability of DTI to track the progression of microstructural damage in patients with inherited ataxias has not been explored so far. We performed a longitudinal DTI study in patients with spinocerebellar ataxia type 2.

MATERIALS AND METHODS: Ten patients with spinocerebellar ataxia type 2 and 16 healthy age-matched controls were examined twice with DTI (mean time between scans, 3.6 years [patients] and 3.3 years [controls]) on the same 1.5T MR scanner. Using tract-based spatial statistics, we analyzed changes in DTI-derived indices: mean diffusivity, axial diffusivity, radial diffusivity, fractional anisotropy, and mode of anisotropy.

RESULTS: At baseline, the patients with spinocerebellar ataxia type 2, as compared with controls, showed numerous WM tracts with significantly increased mean diffusivity, axial diffusivity, and radial diffusivity and decreased fractional anisotropy and mode of anisotropy in the brain stem, cerebellar peduncles, cerebellum, cerebral hemisphere WM, corpus callosum, and thalami. Longitudinal analysis revealed changes in axial diffusivity and mode of anisotropy in patients with spinocerebellar ataxia type 2 that were significantly different than those in the controls. In patients with spinocerebellar ataxia type 2, axial diffusivity was increased in WM tracts of the right cerebral hemisphere and the corpus callosum, and the mode of anisotropy was extensively decreased in hemispheric cerebral WM, corpus callosum, internal capsules, cerebral peduncles, pons and left cerebellar peduncles, and WM of the left paramedian vermis. There was no correlation between the progression of changes in DTI-derived indices and clinical deterioration.
\end{abstract}

CONCLUSIONS: DTI can reveal the progression of microstructural damage of WM fibers in the brains of patients with spinocerebellar ataxia type 2, and mode of anisotropy seems particularly sensitive to such changes. These results support the potential of DTI-derived indices as biomarkers of disease progression.

ABBREVIATIONS: $A D=$ axial diffusivity; FA = fractional anisotropy; $M D=$ mean diffusivity; $M O=$ mode of anisotropy; $R D=$ radial diffusivity; $S C A 2=$ spinocerebellar ataxia type 2; TBSS = tract-based spatial statistics; MNI = Montreal Neurological Institute

S pinocerebellar ataxia type 2 (SCA2) is the second most frequent autosomal dominant inherited ataxia worldwide, after SCA3. ${ }^{1}$ It is caused by expansion in excess of 32 CAG repeats in the gene encoding the Ataxin-2 protein, which mainly targets several

Received July 29, 2014; accepted after revision November 21.

From the Quantitative and Functional Neuroradiology Research Unit (M.M.), Meyer Children and Careggi Hospitals of Florence, Florence, Italy; "Mario Serio" Department of Experimental and Clinical Biomedical Sciences (M.M., A.B.), University of Florence, Florence, Italy; Medical Physics Section (N.T.), Department of Biomedicine and Prevention, University of Rome "Tor Vergata," Rome, Italy; Department of Radiology (N.T.), Athinoula A. Martinos Center for Biomedical Imaging, Boston, Massachusetts; Harvard Medical School (N.T.), Boston, Massachusetts; Unit of Medical Physics (M.G.), Pisa University Hospital “Azienda Ospedaliero-Universitaria Pisana," Pisa, Italy; Neuroradiology Unit (A.G.), Careggi General Hospital, Florence, Italy; "San Giuseppe" Hospital (R.D.N.), Empoli, Italy; IRCSS SDN Foundation (E.N., M.A., A.S.), Naples, Italy; Unit of Radiology (C.T.), Versilia Hospital, Lido di Camaiore, Italy; Department of Neurological Sciences (E.S.), University of Naples Federico II, Naples, Italy; University of Naples Parthenope (A.S.), Naples, Italy; Department of Electrical, Electronic, and Information Engineering "Guglielmo Marconi" (S.D.), University of Bologna, Cesena, Italy. pontine neurons and Purkinje cells in the cerebellum, ${ }^{2}$ and it is associated with a pathologic pattern of pontocerebellar atrophy. ${ }^{1,3}$ MR T1-weighted imaging enables in vivo detection of brain stem and cerebellar atrophy in patients with SCA2 in crosssectional $^{4-6}$ and longitudinal ${ }^{7}$ studies.

Recently, DWI and DTI have enabled quantitative assessment of the microstructural changes in brain tissue that result from neurodegenerative diseases. ${ }^{5,8-21}$ In particular, in longitudinal studies, DTI may be a sensitive instrument for tracking the progression of neurodegeneration (namely, neuronal damage and loss, Wallerian degeneration, demyelination, and gliosis) and, we hope, for detecting the efficacy (or lack of thereof) of new thera-

Please address correspondence to Mario Mascalchi, MD, PhD, "Mario Serio" Department of Experimental and Clinical Biomedical Sciences, University of Florence, Viale Morgagni 50, Florence, Italy; e-mail: m.mascalchi@dfc.unifi.it

Indicates article with supplemental on-line photo.

http://dx.doi.org/10.3174/ajnr.A4343 
Table 1: Demographic, genetic, and clinical data of 10 patients with SCA2

\begin{tabular}{lcccccc}
\hline $\begin{array}{c}\text { Patient } \\
\text { No. }\end{array}$ & Age (yr) & Sex & $\begin{array}{c}\text { No. of Triplet } \\
\text { Repeat Expansions }\end{array}$ & $\begin{array}{c}\text { Disease } \\
\text { Duration (yr) }\end{array}$ & $\begin{array}{c}\text { IACRS at } \\
\text { Baseline }\end{array}$ & $\begin{array}{c}\text { IACRS at } \\
\text { Follow-Up }\end{array}$ \\
\hline 1 & 60.6 & M & 38 & 20 & 20 & 23 \\
2 & 28.4 & M & 39 & 2 & 9 & 14 \\
3 & 31.5 & M & 43 & 7 & 16 & 16 \\
4 & 46.5 & $\mathrm{~F}$ & 41 & 7 & 17 & 21 \\
5 & 47.4 & $\mathrm{~F}$ & 41 & 10 & 19 & 27 \\
6 & 43.7 & $\mathrm{M}$ & 41 & 8 & 15 & 15 \\
7 & 67.8 & $\mathrm{M}$ & 41 & 23 & 14 & 18 \\
8 & 54.5 & $\mathrm{~F}$ & 42 & 23 & 25 & 31 \\
9 & 56.9 & $\mathrm{M}$ & 40 & 14 & 20 & 27 \\
10 & 37.7 & $\mathrm{~F}$ & 40 & 14 & 17 & 21 \\
Mean (SD) & $47.5(12.7)$ & & $40.6(1.4)$ & $12.8(7.3)$ & $17.2(4.3)$ & $21.3(5.7)$ \\
\hline
\end{tabular}

Note:-IACRS indicates Inherited Ataxia Clinical Rating Scale.

peutic strategies. So far, relatively few studies have addressed this point, ${ }^{22-30}$ and none have addressed it in relation to autosomal dominant ataxias.

We performed a longitudinal DTI study in 10 patients with SCA2 and 16 age-matched healthy controls to explore the ability of DTI to detect and map the progression of microstructural damage reflecting advance of neurodegeneration. In particular, we analyzed several DTI-derived indices, including mean diffusivity (MD), axial diffusivity (AD), radial diffusivity (RD), fractional anisotropy (FA), and mode of anisotropy (MO), by using tractbased spatial statistics (TBSS), which enable a robust and unbiased voxelwise whole-brain analysis of the main white matter tracts. ${ }^{19,21,31,32}$

\section{MATERIALS AND METHODS \\ Subjects}

Ten patients ( 4 women, 6 men; mean age, $47.5 \pm 12.7$ years) with a genetically determined diagnosis of SCA $2^{33}$ gave their consent to participate in this longitudinal study, which was approved by the local ethics committee. The cutoff number of triplet repeat expansions that qualified for diagnosis of SCA2 was 34 CAG repeats on 1 allele, and the mean number of abnormal triplets was $40.6 \pm 1.4$. Each patient underwent MR imaging twice on the same scanner and with the same acquisition protocol at an average of $3.6 \pm 0.7$ years apart (range, 2.2-4.1 years). At the time of both MR imaging examinations, each patient underwent evaluation by the same clinician (A.G.), who assessed the neurologic deficit by using the Inherited Ataxia Clinical Rating Scale. ${ }^{34}$ This is a $0-38$ scale ( 38 corresponds to maximum clinical deficit) that evaluates signs and symptoms related to ataxia and also pyramidal tract dysfunction and impaired vibration or position sense, which are frequently observed in SCA2. ${ }^{1}$ At the time of baseline MR imaging, the mean disease duration from clinical onset in patients with SCA2 was $12.8 \pm 7.3$ years (range, $2-23$ years), and the mean Inherited Ataxia Clinical Rating Scale score was $17.2 \pm 4.3$ (range, 9-25). Demographic, genetic, and clinical data of the patients with SCA2 are summarized in Table 1.

Sixteen age- and sex-matched healthy subjects ( 7 women, 9 men; mean age, $50.3 \pm 18.8$ years) were recruited as controls and provided written consent to participate in the study. They had no history of neurologic or psychiatric dysfunction, and results of their neurologic examinations at baseline and follow-up were negative. Control subjects also underwent MR imaging twice with the same scanner and acquisition protocol at an of average of $3.3 \pm 1.0$ years apart (range, $1.9-4.7$ years).

The TBSS results from the baseline examinations of 9 of the patients with SCA2 and 10 of the healthy controls enrolled in the present study were reported previously. ${ }^{19}$

\section{MR Imaging Protocol}

The patients and controls underwent MR imaging in a single center on a $1.5 \mathrm{~T}$ system (Intera; Philips, Best, the Netherlands) with a $33 \mathrm{mT} / \mathrm{m}$ maximum gradient strength. After scout imaging, axial diffusion-weighted images were obtained with single-shot echo-planar imaging sequences (TR, 9394 ms; TE, 89 ms; FOV, $256 \times 256$ $\mathrm{mm}$; matrix size, $128 \times 128$; number of sections, 50 ; section thickness, $3 \mathrm{~mm}$; no intersection gap; number of excitations, 3 ; sensitivity encoding acceleration factor, 2) by using a 6-channel head coil. Diffusion-sensitizing gradients were applied along 15 noncollinear directions by using a b-value of 0 (B0 image) and $1000 \mathrm{~s} / \mathrm{mm}^{2}$. As part of the protocol, we also acquired sagittal 3D T1-weighted turbo gradient-echo images (TR, $8.1 \mathrm{~ms}$; TE, $3.7 \mathrm{~ms}$; flip angle, $8^{\circ}$; inversion time, $764 \mathrm{~ms}$; FOV, $256 \times 256 \mathrm{~mm}$; matrix size, $256 \times 256$; contiguous sections, 160; section thickness, $1 \mathrm{~mm}$ ). T1-weighted images were used in a longitudinal morphometry study. ${ }^{7}$

\section{Image Processing and TBSS}

Preliminarily visual evaluations of all B0 and diffusion-weighted images revealed no motion artifacts in any patient with SCA2 or control subject. Diffusion-weighted images were corrected for head motion and eddy current distortions by using the FMRIB Diffusion Toolbox (2.0; http://www.fmrib.ox.ac.uk/fsl/fdt/index. html), part of the FMRIB Software Library (FSL 5.0.2; http:// www.fmrib.ox.ac.uk/fsl), ${ }^{35}$ after which brain tissue was segmented by using the FSL Brain Extraction Tool (http://fsl.fmrib. ox.ac.uk/fsl/fslwiki/BET). The b-matrix was subsequently reoriented by applying the rotational part of the affine transformation used in the eddy/motion-correction step. ${ }^{36}$ A tensor model was then fitted to the raw data by using a constrained nonlinear leastsquares procedure implemented in the software package Camino (www.camino.org.uk), ${ }^{37}$ and residual nonpositive definite tensors (in regions in which the nonlinear algorithm failed to converge) were removed by tensor interpolation in the log-Euclidean domain. ${ }^{38}$ Diffusion tensor invariants, $\mathrm{MD}, \mathrm{AD}, \mathrm{RD}, \mathrm{FA}$, and MO, were then computed from the estimated tensor field by using the DTI ToolKit software package (http://software.incf.org/software/dtitoolkit-dti-tk). ${ }^{39}$ The MO is an adimensional tensor invariant (range, -1 to 1 ) that characterizes anisotropy type. ${ }^{40}$ In particular, the MO specifies the type of anisotropy as a continuous measure reflecting differences in the shape of the diffusion tensor ranging from planar (eg, in regions of crossing fibers from 2 fiber populations of similar attenuation or regions of "kissing" fibers [low MO value]) to linear (eg, in regions in which 1 fiber population orientation predominates [high MO value]). ${ }^{41}$

Voxelwise statistical analyses of all tensor invariant data were carried out by using TBSS, ${ }^{32}$ also part of the FMRIB Software Library, 
which involves 1) nonlinear registration of every FA image to every other one, 2) identification of the "most representative image" as the one that requires the least warping to align every other image to it, 3 ) affine alignment of this target image into Montreal Neurological Institute 152 (MNI152) space, 4) transformation of every other image into MNI space by combining the single nonlinear transforms into the target image with the affine transform into MNI space, 5) creation of a mean FA image, and 6) thinning to create a mean FA skeleton that represents the centers of all tracts common to the group. Each subject's aligned FA data were then projected onto this skeleton, and previously computed warps were also successively applied to $\mathrm{MD}, \mathrm{AD}, \mathrm{RD}$, and $\mathrm{MO}$ images, thereby transforming all images into MNI space for subsequent skeletonization as described above. The resulting data were then fed into voxelwise intersubject and intrasubject statistics. Each analysis included full correction for multiple comparisons over space by using permutation-based nonparametric inference within the framework of the general linear model. Specifically, a first general linear model included only group ("between" factor) to investigate the effect of group (patients versus controls) at baseline. A second general linear model included both group and time ("within" factor), which enabled us to investigate the grouptime interaction (patient versus control differences in follow-up versus baseline changes). In another general linear model, we investigated within-patient correlation between changes in DTI-derived indices and changes of clinical variables. All the general linear model designs included age and sex as nuisance covariates. Also, with the aim of taking into account potential confounders resulting from longitudinal atrophy, ${ }^{7}$ every subject's follow-up T1 scan was registered to the corresponding baseline T1 scan by using high-dimensional nonlinear registration, after which a Jacobian determinant image (which quantifies regional volume change) was computed for the inverse transformations. The baseline scans were all registered to a population-specific $\mathrm{T} 1$ template, which in turn was registered to the MNI152 T1 template. The Jacobian determinant image was then transformed into MNI152 space through a single interpolation step in which all the aforementioned transformations were combined. Full details about this procedure can be found in Mascalchi et $\mathrm{al}^{7}$ and Tessa et al. ${ }^{42}$ After masking out CSF, the whole-brain average of the Jacobian determinant image was inserted into the longitudinal general linear models as a subject-specific nuisance covariate of global atrophy.

To improve the confidence intervals on resulting $P$-value maps, in each comparison we used 50,000 permutations (as opposed to the commonly used value of 5000). $P$ values were calculated and corrected for multiple comparisons by using the $2 \mathrm{D}$ parameter settings with threshold-free cluster enhancement, thereby avoiding the use of an arbitrary threshold for the initial cluster formation. ${ }^{43}$

\section{RESULTS}

\section{Baseline Evaluations}

TBSS showed changes in all DTI-derived indices in several WM tracts of the brain in patients with SCA2 that were significant compared with those of the controls. These tracts included the cerebral and cerebellar WM, the corpus callosum, brain stem, cerebellar peduncle, and thalamus (On-line Fig 1) and consisted of increased MD and RD and decreased FA and MO. AD was increased in the brain stem, cerebellum, and cerebellar peduncles, and the WM was underlying the right primary sensorimotor cortex, corpus callosum, and thalami. However, $\mathrm{AD}$ was decreased in the right internal capsule and frontal WM. There were no significant decreases in $\mathrm{MD}$ or $\mathrm{RD}$ or significant increases in FA or MO.

\section{Longitudinal Evaluations}

TBSS revealed longitudinal $\mathrm{AD}$ and $\mathrm{MO}$ changes in patients with SCA2 that were significant compared with those of the controls (Fig 1).

Longitudinal AD changes were significantly greater (ie, increased) in patients with SCA2 than in controls in the right cerebral hemisphere and corpus callosum (Fig 1). The mean longitudinal changes in these regions are listed in Table 2.

Longitudinal MO changes were significantly lower (ie, decreased) in patients with SCA2 than in controls in many WM tracts, including bilateral hemispheric cerebral WM, corpus callosum, internal capsule, cerebral peduncle, pons, and left inferior, middle, and superior cerebellar peduncle tracts and in the WM of the left paramedian vermis (Fig 1). The mean longitudinal changes in these regions are listed in Table 2.

No WM tract showed significant differences in longitudinal $\mathrm{MD}, \mathrm{RD}$, or FA changes in patients with SCA2 versus those in controls.

\section{MR Imaging Clinical Correlations}

No significant correlations between clinical progression (modification of the Inherited Ataxia Clinical Rating Scale) and changes in the DTI-derived indices were observed.

\section{DISCUSSION}

In patients with SCA2, histopathologic studies have shown widespread neuronal loss, which is prominent in the cerebellar cortex and pontine nuclei, midbrain, medulla, and motor cortex. ${ }^{1,3,44}$ WM damage in patients with SCA2 consists of a loss of myelinated fibers and gliosis affecting the transverse pontine fibers, central cerebellum and cerebellar folia, middle and inferior cerebellar peduncles, medial lemniscus and trigeminal tracts, fasciculus gracilis and cuneatus, and spinocerebellar tracts. ${ }^{1}$ Gliosis has been observed also in the thalamus, globus pallidus, and subthalamic region. ${ }^{1}$

MR imaging shows loss of bulk of the brain stem and cerebellum in presymptomatic and early symptomatic SCA2 gene carri$\mathrm{ers}^{4-6}$ and circumscribed atrophy of the frontal, parietal, and temporal cortex, the thalamus, and frontal and temporal WM in more advanced cases. ${ }^{19,45,46}$ MR imaging can also show diffuse high signal intensity of the brain stem and cerebellar WM with sparing of the corticospinal tracts in proton-attenuation and T2-weighted images. ${ }^{47}$ Two studies evaluated patients with SCA2 with DTI using regions of interest ${ }^{16}$ or MD histogram analysis and TBSS. ${ }^{19}$ Both studies revealed a widespread decrease in FA and an increase in the apparent diffusion coefficient, $\mathrm{MD}$, and $\mathrm{RD}$, which are consistent with microstructural damage of WM tracts in the brain stem, cerebellar peduncles, and cerebellum and in the corpus callosum and several WM tracts in the cerebral hemispheres, including the corticospinal tracts, inferior longitudinal fasciculus, and inferior fronto-occipital fasciculus.

To the best of our knowledge, this is the first DTI study to have explored longitudinal changes of the brain regional microstructure in patients with SCA2 compared with those of healthy age-matched 


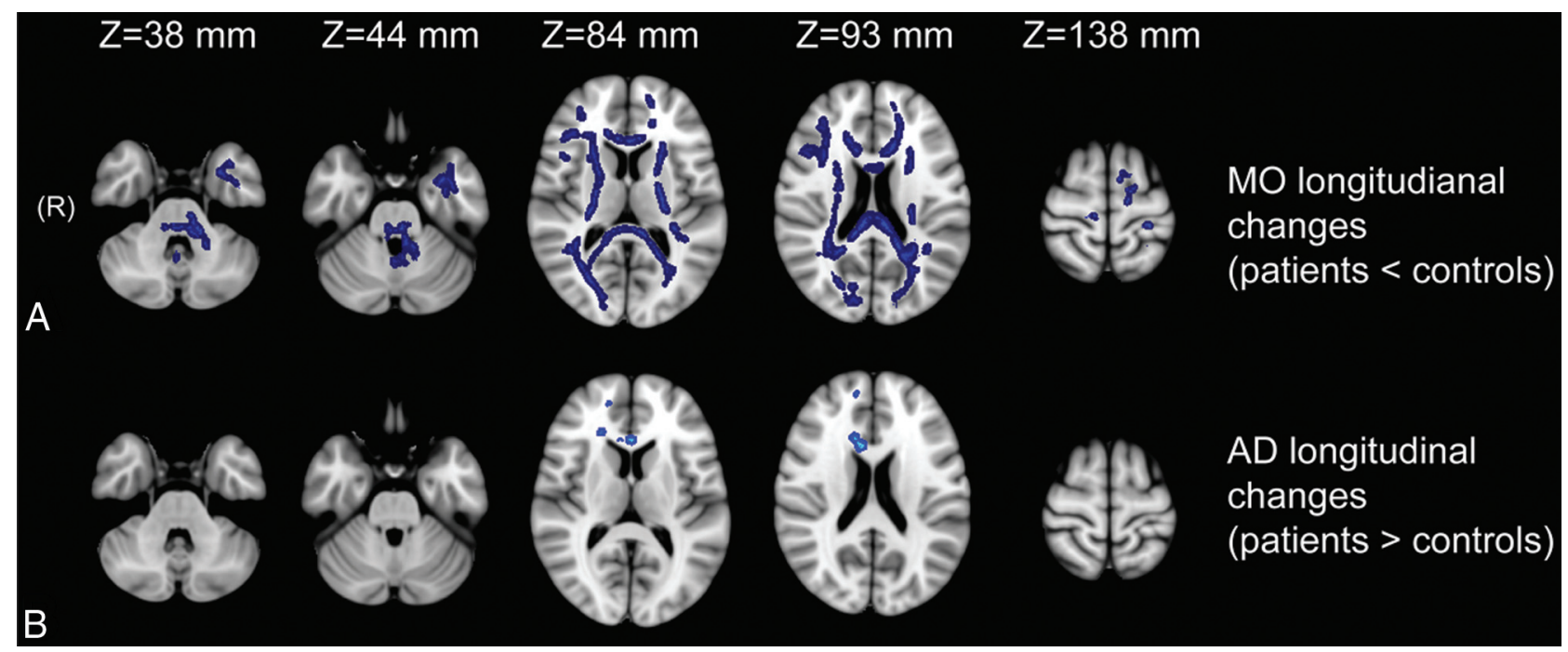

FIG 1. Longitudinal between-group TBSS analyses at different anatomic levels (z coordinate in Montreal Neurological Institute 152 space). TBSS revealed differences in longitudinal changes of some DTI-derived indices (AD and $M O$ ) in WM tracts in patients with SCA2 that were significant compared with those of the controls. A, Longitudinal MO changes were significantly greater in patients with SCA2 than in controls and extensively involved hemispheric cerebral WM, corpus callosum, internal capsules, cerebral peduncles, pons, and left cerebellar peduncles. Longitudinal $A D$ changes were significantly greater in the right cerebral hemisphere and the corpus callosum of the patients with SCA2 than in the controls $(B)$.

Table 2: Mean values of DTI-derived indices in regions in which the longitudinal AD and MO changes were significantly different between patients with SCA2 and controls

\begin{tabular}{lcccccccc} 
& \multicolumn{3}{c}{ Patients with SCA2 } & & \multicolumn{3}{c}{ Controls } \\
\cline { 2 - 4 } DTI-Derived Index & $\begin{array}{c}\text { Mean Value at } \\
\text { Baseline }\end{array}$ & $\begin{array}{c}\text { Mean Value at } \\
\text { Follow-Up }\end{array}$ & $\begin{array}{c}\text { Mean Value Change } \\
\text { (Follow-Up - Baseline) }\end{array}$ & $\begin{array}{c}\text { Mean Value at } \\
\text { Baseline }\end{array}$ & $\begin{array}{c}\text { Mean Value at } \\
\text { Follow-Up }\end{array}$ & $\begin{array}{c}\text { Mean Value Change } \\
\text { (Follow-Up - Baseline) }\end{array}$ \\
\hline $\mathrm{AD}\left(\times 10^{-3} \mathrm{~mm}^{2} / \mathrm{s}\right)$ & 1.17 & 1.28 & 0.11 & & 1.18 & 1.16 & -0.02 \\
$\mathrm{MO}$ & 0.51 & 0.37 & -0.14 & & 0.56 & 0.62 & 0.06 \\
\hline
\end{tabular}

controls. In particular, patients with SCA2 showed progression of WM damage in the hemispheric cerebral WM, corpus callosum, pons, and left cerebellar peduncles and in the WM of the left paramedian vermis. We noted a hemispheric asymmetry of some longitudinal DTI changes in our patients with SCA2, which may reflect either the relatively low sample size or a truly uneven distribution of the neurodegenerative process. Asymmetry of damage was previously reported from MR imaging studies of patients with Huntington disease, familial Alzheimer disease, and Friedreich ataxia ${ }^{21,48-51}$ and is not uncommon in pathologic studies of neurodegenerative diseases. ${ }^{52}$ In our opinion, the issue of the symmetric versus asymmetric distribution of microstructural changes revealed by DTI needs further investigation with larger sample sizes.

We believe that a thorough analysis of the cross-sectional and longitudinal changes of the various DTI-derived indices that were used in this study, and their interplay in a typical neurodegenerative disease such as SCA2, can be of interest. At baseline, TBSS showed widespread changes in DTI-derived indices in patients with SCA2 compared with those in the controls. These changes include increases in $\mathrm{MD}$ and $\mathrm{RD}$ and decreases in FA and $\mathrm{MO}$. $\mathrm{AD}$ was increased in the brain stem, cerebellum and cerebellar peduncles, thalami, and WM underlying the (right) primary sensory cortex and decreased in the right internal capsule and frontal WM. The increases in MD and RD and decreases in FA are in line with those reported so far in cross-sectional studies of various neurodegenerative diseases. ${ }^{5,8-11,13-25,27-29,53}$ In our study, we observed areas of increased $\mathrm{AD}$ that are in line with those shown in cross-sectional studies of patients with Alzheimer disease, ${ }^{53-56}$ Friedreich ataxia, ${ }^{20}$ and Huntington disease. ${ }^{13,55}$ It is notable that an increase in $\mathrm{AD}$ per se is not inconsistent with a decrease in FA. In fact, it is sufficient for the increased $\mathrm{AD}$ to be accompanied by a more pronounced increase in $\mathrm{RD}$ to result in a decrease of FA in degenerating WM fibers. ${ }^{20}$ Also, we observed an area of decreased $\mathrm{AD}$ in the right internal capsule and frontal WM. The coexistence of WM areas exhibiting increased and decreased $\mathrm{AD}$ was reported from a study of Alzheimer disease ${ }^{53}$ and supports the view that AD may show time-dependent behavior related to a variable combination of factors determining either a decrease caused by Wallerian degeneration and gliosis or an increase caused by axonal damage and atrophy of WM fiber tracts with expanded extracellular space. In our opinion, the complex interplay of these processes should be addressed in animal-model studies. ${ }^{57}$

To our knowledge, so far, only 1 cross-sectional study in patients with mild cognitive impairment and mild Alzheimer disease addressed MO changes in patients with a neurodegenerative disease. ${ }^{41}$ An initially counterintuitive increase in the MO was observed in a region of crossing fibers (ie, planar anisotropy) in the centrum semiovale of patients with mild cognitive impairment versus controls and was explained by selective preservation of motor-related projection fibers (at an early stage of the disease) crossing the degenerating association fibers of the superior longitudinal fasciculus, which results in more linear anisotropy. ${ }^{41}$ In our study, we observed at baseline a widespread decrease of the MO in patients with SCA2 versus that of controls, which included areas with crossing fibers such as cerebellar 
hemispheric WM, centrum semiovale, lobar WM, and areas without crossing fibers such as the cerebellar peduncles, brain stem longitudinal tracts, external and internal capsules, and corpus callosum. Given that a decrease in the MO indicates a reduction of the degree of linear anisotropy, our results suggest a general disorganization of WM fibers in patients with SCA2.

The longitudinal data in our study reveal significantly more pronounced modifications of $\mathrm{AD}$ and $\mathrm{MO}$ in patients with SCA2 than in the controls. In the patients with SCA2, the increase in AD was circumscribed to the right frontal WM and corpus callosum, whereas the decrease in the MO involved symmetrically and extensively the hemispheric cerebral WM and internal capsules, the corpus callous, the cerebral peduncles, the pons, all 3 of the cerebellar peduncles, and the left paramedian vermis WM. No areas of significant difference in changes in $\mathrm{MD}, \mathrm{RD}$, or $\mathrm{FA}$ in patients with SCA2 were detected compared with those in controls.

The increase in $\mathrm{AD}$ at follow-up in the patients with SCA2 is in line with the data from a longitudinal study of symptomatic patients with Huntington disease and was interpreted as the reflection of increased extra-axonal space, resulting from reduced axonal caliber, which allows faster water-molecule movement parallel to axons. ${ }^{55}$ The decrease in the MO at follow-up in patients with SCA2 is in line with the progressive WM fiber disorganization expected in a neurodegenerative disease. It is notable that the far more extensive longitudinal changes in the MO compared with the modifications in $\mathrm{AD}$, including WM tracts in the brain stem and cerebellum, may support the potential use of the $\mathrm{MO}$ as an additional valuable and sensitive descriptor of microstructural changes. ${ }^{41}$ We submit the following non-mutually exclusive explanations for the lack of significant differences in longitudinal MD, FA, and RD modification in our group of patients with SCA2 compared with those in the control group: 1) the occurrence of a "ceiling" effect; 2 ) low statistical power ( $\beta$ error) (in this regard, a gain in the signal-to-noise ratio, which can be obtained at higher field strength [eg, 3T] or by using a head coil with a greater number of channels [eg, 32] for advanced neuroimaging) and the use of high angular resolution diffusion-weighted imaging acquisitions would have improved the sensitivity of our study in detecting any white matter changes in the brain stem and/or cerebellum.

Finally, we preliminarily explored whether longitudinal DTI changes were correlated with clinical progression in patients with SCA2. The lack of a significant correlation between longitudinal DTI changes and clinical deterioration in our study (which has been observed also for morphometry findings in patients with SCA $2^{7}$ and those with SCA1, SCA3, or SCA $6^{58}$ ) might reflect the small sample size, but in our opinion, supports the role of DTI metrics as potential biomarkers of the progression of disease-related microstructural alterations that may precede the evolution of clinical disease manifestations.

However, we did not obtain a structured assessment of cognitive or behavioral changes in our patients. We therefore could not explore the possibility that DTI modifications are correlated with the evolution of nonmotor features. Given that a recent study indicated that a simple clinical examination can track disease progression in paucisymptomatic SCA2 gene carriers, ${ }^{59}$ we feel that a tight comparative evaluation of clinical and MR techniques including DTI and morphometry in monitoring neurodegenera- tion is advisable for ascertaining the optimal strategy in view of future trials, especially in patients in the early stages of disease. ${ }^{60}$

\section{CONCLUSIONS}

DTI is capable of tracking the progression of microstructural brain damage and therefore represents a candidate biomarker of the progression of neurodegeneration in patients with SCA2.

\section{REFERENCES}

1. Auburger GW. Spinocerebellar ataxia type 2. Handb Clin Neurol 2012;103:423-36

2. Orr HT. Cell biology of spinocerebellar ataxia. J Cell Biol 2012;197:167-77

3. Seidel K, Siswanto S, Brunt ER, et al. Brain pathology of spinocerebellar ataxias. Acta Neuropathol 2012;124:1-21

4. Jacobi H, Reetz K, du Montcel ST, et al. Biological and clinical characteristics of individuals at risk for spinocerebellar ataxia types 1,2 , 3 , and 6 in the longitudinal RISCA study: analysis of baseline data. Lancet Neurol 2013;12:650-58

5. Mascalchi M, Vella A. Magnetic resonance and nuclear medicine imaging in ataxias. Handb Clin Neurol 2012;103:85-110

6. Jacobi H, Hauser TK, Giunti P, et al. Spinocerebellar ataxia types 1, 2, 3 and 6: the clinical spectrum of ataxia and morphometric brainstem and cerebellar findings. Cerebellum 2012;11:155-66

7. Mascalchi M, Diciotti S, Giannelli M, et al. Progression of brain atrophy in spinocerebellar ataxia type 2: a longitudinal tensor-based morphometry study. PLoS One 2014;9:e89410

8. Agosta F, Pievani M, Sala S, et al. White matter damage in Alzheimer disease and its relationship to gray matter atrophy. Radiology 2011;258:853-63

9. Teipel SJ, Grothe M, Lista S, et al. Relevance of magnetic resonance imaging for early detection and diagnosis of Alzheimer disease. Med Clin North Am 2013;97:399-424

10. Zhang Y, Tartaglia MC, Schuff N, et al. MRI signatures of brain macrostructural atrophy and microstructural degradation in frontotemporal lobar degeneration subtypes. J Alzheimers Dis 2013;33:431-44

11. Mascalchi M, Vella A, Ceravolo R. Movement disorders: role of imaging in diagnosis. J Magn Reson Imaging 2012;35:239-56

12. Tessa C, Giannelli M, Della Nave R, et al. A whole-brain analysis in de novo Parkinson disease. AJNR Am J Neuroradiol 2008;29:674-80

13. Della Nave R, Ginestroni A, Tessa C, et al. Regional distribution and clinical correlates of white matter structural damage in Huntington disease: a tract-based spatial statistics study. AJNR Am J Neuroradiol 2010;31:1675-81

14. Cosottini M, Giannelli M, Siciliano G, et al. Diffusion-tensor MR imaging of corticospinal tract in amyotrophic lateral sclerosis and progressive muscular atrophy. Radiology 2005;237:258-64

15. Guimaraes RP, D'Abreu A, Yasuda CL, et al. A multimodal evaluation of microstructural white matter damage in spinocerebellar ataxia type 3. Mov Disord 2013;28:1125-32

16. Mandelli ML, De Simone T, Minati L, et al. Diffusion tensor imaging of spinocerebellar ataxias types 1 and 2. AJNR Am J Neuroradiol 2007;28:1996-2000

17. Salvatore E, Tedeschi E, Mollica C, et al. Supratentorial and infratentorial damage in spinocerebellar ataxia 2: a diffusion-weighted MRI study. Mov Disord 2014;29:780-86

18. Kang JS, Klein JC, Baudrexel S, et al. White matter damage is related to ataxia severity in SCA3. J Neurol 2014;261:291-99

19. Della Nave R, Ginestroni A, Tessa C, et al. Brain white matter damage in SCA1 and SCA2. An in vivo study using voxel-based morphometry, histogram analysis of mean diffusivity and tract-based spatial statistics. Neuroimage 2008;43:10-19

20. Della Nave R, Ginestroni A, Diciotti S, et al. Axial diffusivity is increased in the degenerating superior cerebellar peduncles of Friedreich's ataxia. Neuroradiology 2011;53:367-72

21. Della Nave R, Ginestroni A, Tessa C, et al. Brain white matter tracts 
degeneration in Friedreich ataxia. An in vivo MRI study using tractbased spatial statistics and voxel-based morphometry. Neuroimage 2008;40:19-25

22. Amlien IK, Fjell AM, Walhovd KB, et al. Mild cognitive impairment: cerebrospinal fluid tau biomarker pathologic levels and longitudinal changes in white matter integrity. Radiology 2013;266:295-303

23. Keil C, Prell T, Peschel T, et al. Longitudinal diffusion tensor imaging in amyotrophic lateral sclerosis. BMC Neurosci 2012;13:141

24. Pellecchia MT, Barone P, Vicidomini C, et al. Progression of striatal and extrastriatal degeneration in multiple system atrophy: a longitudinal diffusion-weighted MR study. Mov Disord 2011;26:1303-09

25. Reginold W, Lang AE, Marras C, et al. Longitudinal quantitative MRI in multiple system atrophy and progressive supranuclear palsy. Parkinsonism Relat Disord 2014;20:222-25

26. Teipel SJ, Meindl T, Wagner M, et al. Longitudinal changes in fiber tract integrity in healthy aging and mild cognitive impairment: a DTI follow-up study. J Alzheimers Dis 2010;22:507-22

27. Vandenberghe W, Demaerel P, Dom R, et al. Diffusion-weighted versus volumetric imaging of the striatum in early symptomatic Huntington disease. J Neurol 2009;256:109-14

28. Weaver KE, Richards TL, Liang O, et al. Longitudinal diffusion tensor imaging in Huntington's disease. Exp Neurol 2009;216:525-29

29. Sritharan A, Egan GF, Johnston L, et al. A longitudinal diffusion tensor imaging study in symptomatic Huntington's disease. J Neurol Neurosurg Psychiatry 2010;81:257-62

30. Seppi K, Schocke MF, Mair KJ, et al. Progression of putaminal degeneration in multiple system atrophy: a serial diffusion MR study. Neuroimage 2006;31:240-45

31. Smith SM, Johansen-Berg H, Jenkinson M, et al. Acquisition and voxelwise analysis of multi-subject diffusion data with tract-based spatial statistics. Nat Protoc 2007;2:499-503

32. Smith SM, Jenkinson M, Johansen-Berg H, et al. Tract-based spatial statistics: voxelwise analysis of multi-subject diffusion data. Neuroimage 2006;31:1487-505

33. Pareyson D, Gellera C, Castellotti B, et al. Clinical and molecular studies of 73 Italian families with autosomal dominant cerebellar ataxia type I: SCA1 and SCA2 are the most common genotypes. J Neurol 1999;246:389-93

34. Filla A, DeMichele G, Caruso G, et al. Genetic data and natural history of Friedreich's disease: a study of $\mathbf{8 0}$ Italian patients. J Neurol 1990;237:345-51

35. Smith SM, Jenkinson M, Woolrich MW, et al. Advances in functional and structural MR image analysis and implementation as FSL. Neuroimage 2004;23(suppl 1):S208-19

36. Leemans A, Jones DK. The B-matrix must be rotated when correcting for subject motion in DTI data. Magn Reson Med 2009;61:1336-49

37. Cook PA, Bai Y, Nedjati-Gilani S, et al. Camino: open-source diffusion-MRI reconstruction and processing. In: Proceedings of the 14th Scientific Meeting of the International Society for Magnetic Resonance in Medicine, Seattle, Washington. May 6-12, 2006:2759

38. Arsigny V, Fillard P, Pennec X, et al. Log-Euclidean metrics for fast and simple calculus on diffusion tensors. Magn Reson Med 2006;56:411-21

39. Zhang H, Avants BB, Yushkevich PA, et al. High-dimensional spatial normalization of diffusion tensor images improves the detection of white matter differences: an example study using amyotrophic lateral sclerosis. IEEE Trans Med Imaging 2007;26:1585-97

40. Ennis DB, Kindlmann G. Orthogonal tensor invariants and the analysis of diffusion tensor magnetic resonance images. Magn Reson Med 2006;55:136-46

41. Douaud G, Jbabdi S, Behrens TE, et al. DTI measures in crossing- fibre areas: increased diffusion anisotropy reveals early white matter alteration in MCI and mild Alzheimer's disease. Neuroimage 2011;55:880-90

42. Tessa C, Lucetti C, Giannelli M, et al. Progression of brain atrophy in the early stages of Parkinson's disease: a longitudinal tensor-based morphometry study in de novo patients without cognitive impairment. Hum Brain Mapp 2014;35:3932-44

43. Smith SM, Nichols TE. Threshold-free cluster enhancement: addressing problems of smoothing, threshold dependence and localisation in cluster inference. Neuroimage 2009;44:83-98

44. Rüb U, Seidel K, Ozerden I, et al. Consistent affection of the central somatosensory system in spinocerebellar ataxia type 2 and type 3 and its significance for clinical symptoms and rehabilitative therapy. Brain Res Rev 2007;53:235-49

45. Brenneis C, Bosch SM, Schocke M, et al. Atrophy pattern in SCA2 determined by voxel-based morphometry. Neuroreport 2003;14:1799-802

46. Goel G, Pal PK, Ravishankar S, et al. Gray matter volume deficits in spinocerebellar ataxia: an optimized voxel based morphometric study. Parkinsonism Relat Disord 2011;17:521-27

47. Lee YC, Liu CS, Wu HM, et al. The "hot cross bun" sign in the patients with spinocerebellar ataxia. Eur J Neurol 2009;16:513-16

48. Rosas HD, Liu AK, Hersch S, et al. Regional and progressive thinning of the cortical ribbon in Huntington's disease. Neurology 2002;58:695-701

49. Kipps CM, Duggins AJ, Mahant N, et al. Progression of structural neuropathology in preclinical Huntington's disease: a tensor based morphometry study. J Neurol Neurosurg Psychiatry 2005;76:650-55

50. Thieben MJ, Duggins AJ, Good CD, et al. The distribution of structural neuropathology in pre-clinical Huntington's disease. Brain 2002;125:1815-28

51. Ginestroni A, Battaglini M, Della Nave R, et al. Early structural changes in individuals at risk of familial Alzheimer's disease: a volumetry and magnetization transfer MR imaging study. J Neurol 2009;256:925-32

52. Stefanits H, Budka H, Kovacs GG. Asymmetry of neurodegenerative disease-related pathologies: a cautionary note. Acta Neuropathol 2012;123:449-52

53. Huang $\mathrm{H}$, Fan $\mathrm{X}$, Weiner M, et al. Distinctive disruption patterns of white matter tracts in Alzheimer's disease with full diffusion tensor characterization. Neurobiol Aging 2012;33:2029-45

54. Acosta-Cabronero J, Williams GB, Pengas G, et al. Absolute diffusivities define the landscape of white matter degeneration in Alzheimer's disease. Brain 2010;133:529-39

55. Rosas HD, Lee SY, Bender AC, et al. Altered white matter microstructure in the corpus callosum in Huntington's disease: implications for cortical “disconnection." Neuroimage 2010;49:2995-3004

56. Salat DH, Tuch DS, van der Kouwe AJ, et al. White matter pathology isolates the hippocampal formation in Alzheimer's disease. Neurobiol Aging 2010;31:244-56

57. Kelp A, Koeppen AH, Petrasch-Parwez E, et al. A novel transgenic rat model for spinocerebellar ataxia type 17 recapitulates neuropathological changes and supplies in vivo imaging biomarkers. J Neurosci 2013;33:9068-81

58. Reetz K, Costa AS, Mirzazade S, et al. Genotype-specific patterns of atrophy progression are more sensitive than clinical decline in SCA1, SCA3 and SCA6. Brain 2013;136:905-17

59. Velazquez-Perez L, Rodriguez-Labrada R, Canales-Ochoa N, et al. Progression of early features of spinocerebellar ataxia type 2 in individuals at risk: a longitudinal study. Lancet Neurol 2014;13:482-89

60. Klockgether T. Spinocerebellar ataxia type 2: progression before diagnosis. Lancet Neurol 2014;13:445-46 